\title{
Grain Boundary Segregation and Core/Shell Structured Nanofeatures in Oxide- Dispersion Strengthened Fe-Cr Alloys
}

\author{
Jae-Bok Seol ${ }^{1}$, and Jeoung-Han $\mathrm{Kim}^{2, *}$ \\ 1. National Institute for Nano-materials Technology (NINT), Pohang University of Science and \\ Technology (POSTECH), Pohang, South Korea. \\ 2. Department of Advanced Materials Engineering, Hanbat National University, Daejeon, South Korea. \\ *. Corresponding author: J.-H. Kim
}

Since the oxygen-rich nanofeatures (NFs) in oxide-dispersion-strengthened (ODS) ferritic steels is a key factor for improving their whole mechanical properties [1-3], atom-probe tomography (APT) and transmission electron microscopy (TEM) have been widely employed to identify the oxygen-rich NFs in the materials [2-5]. The current work presents three challenges to deeply understand the chemical origin of the nano-sized precipitates containing Y, O, and Ti. First, the segregation of alloying elements at grain boundaries is observed. Secondly, we compare the concentrations of nano-sized precipitates with a size of $\sim 3 \mathrm{~nm}$ to those of $10-\mathrm{nm}$ precipitates. Finally, the existence of NF structure with the Y-rich cores and Ti- and Cr-enriched shells is confirmed as previously reported by APT. The nominal composition of the materials studied here is $14 \mathrm{Cr}, 3.0 \mathrm{~W}, 0.4 \mathrm{Ti}$ in wt.\%. Analyses were performed using a LEAP-4000HR microscope in voltage-pulsing mode at $200 \mathrm{kHz}$ pulse repetition rate, 0.005 atom/pulse detection rate, $20 \%$ pulse fraction and $70 \mathrm{~K}$.

Fig. 1 shows reconstructed APT maps (a) of alloying solutes in ODS Ti-containing ferritic steels. We found that two grain boundaries are observed as indicated by the arrows. In particular, the $\mathrm{Cr}, \mathrm{W}$, and $\mathrm{Ti}$ atoms have high tendency to segregate to grain boundaries. Nevertheless, the other elements, especially carbon and oxygen atoms, are not detected to segregate at grain boundary. From the cylinder method and iso-surface analyzes, the $1 \mathrm{D}$ concentration profiles of $\mathrm{Cr}, \mathrm{W}$, and $\mathrm{Ti}$ are displayed in Fig.1(b). Note that the profiles were obtained by excluding the NF regions found in the grain boundary. The resulting concentrations of $\mathrm{Cr}, \mathrm{W}$, and $\mathrm{Ti}$ are estimated to be $27.9,3.8$, and 0.9 at.\%, respectively. $\mathrm{The} \mathrm{Cr}$ segregation is observed that extends slightly and shifts underneath the grain boundary further in the analysis direction than the segregation of $\mathrm{W}$ and Ti. This could be explained as being an evaporation artifact due to chromatic aberrations whereby atomic ion trajectory as described in previous report [5].

Fig. 2(a) shows the reconstructed maps of tip\#2 for the steels. The grain boundary segregation of $\mathrm{Cr}, \mathrm{W}$, and $\mathrm{Ti}$ is also detected and the corresponding concentrations are 26.5 for $\mathrm{Cr}, 4.0$ for $\mathrm{W}$, and 1.2 at.\% for Ti, respectively. As the above-mentioned, carbon and oxygen atoms are not segregate at grain boundary. The carbon and oxygen atoms are only partitioned to large NFs with a size of $\sim 10 \mathrm{~nm}$ as well as metal ions $(\mathrm{Al}, \mathrm{Cr})$ and metal-oxides (CrO, YO, and TiO). In smaller NFs with a size of below $5 \mathrm{~nm}$, only $\mathrm{Y}$, $\mathrm{Ti}, \mathrm{O}$, and $\mathrm{TiO}$ ions are detected. This implies that the ratio of Y to Ti in NFs profoundly depends on the size of NFs. In addition, we found that the oxygen atoms exhibit wider lateral extent than Ti ions in NFs. This is explained by evaporation artifact of APT as described by Emmanuelle et al [6]. Besides the oxygen, the $\mathrm{Y}$ ions in all reconstructed particles appear more compressed in the depth direction of NFs than Ti and O ions, as shown in Fig. 2(b). Despite this evaporation artifact of APT, we can determine the concentration ratio of Y to Ti in the NFs through the choice of the sphere ROI including the regions of Y ions detected inside the NFs. As a result, the concentration ratio of $\mathrm{Y}$ to Ti in the NFs varies with the size of NFs. From our results, Ti contributes to the formation of large NFs whereas Y for small and large NFs. We suggest 
here that $\mathrm{Y}$ might participate during the early stages of precipitation to reduce the lattice misfit between NFs and ferrite, thereby make NF nucleation easier. Detailed mechanisms responsible for the formation of NFs will be scientifically discussed.

\section{References:}

[1] M.J. Alinger, G.R. Odette, D.T. Hoelzer, Acta Mater., 57 (2009), p. 392.

[2] M.K. Miller et al, Mater. Sci. Eng. A, 353 (2003), p. 140.

[3] J.H. Kim et al, J. Alloys Compd. 651 (2015), p. 363.

[4] E.A. Marquis, Appl. Phys. Lett. 93 (2008), 181904.

[5] E.A. Marquis, F. Vurpillot, Microsc. Microanal, (to be published).
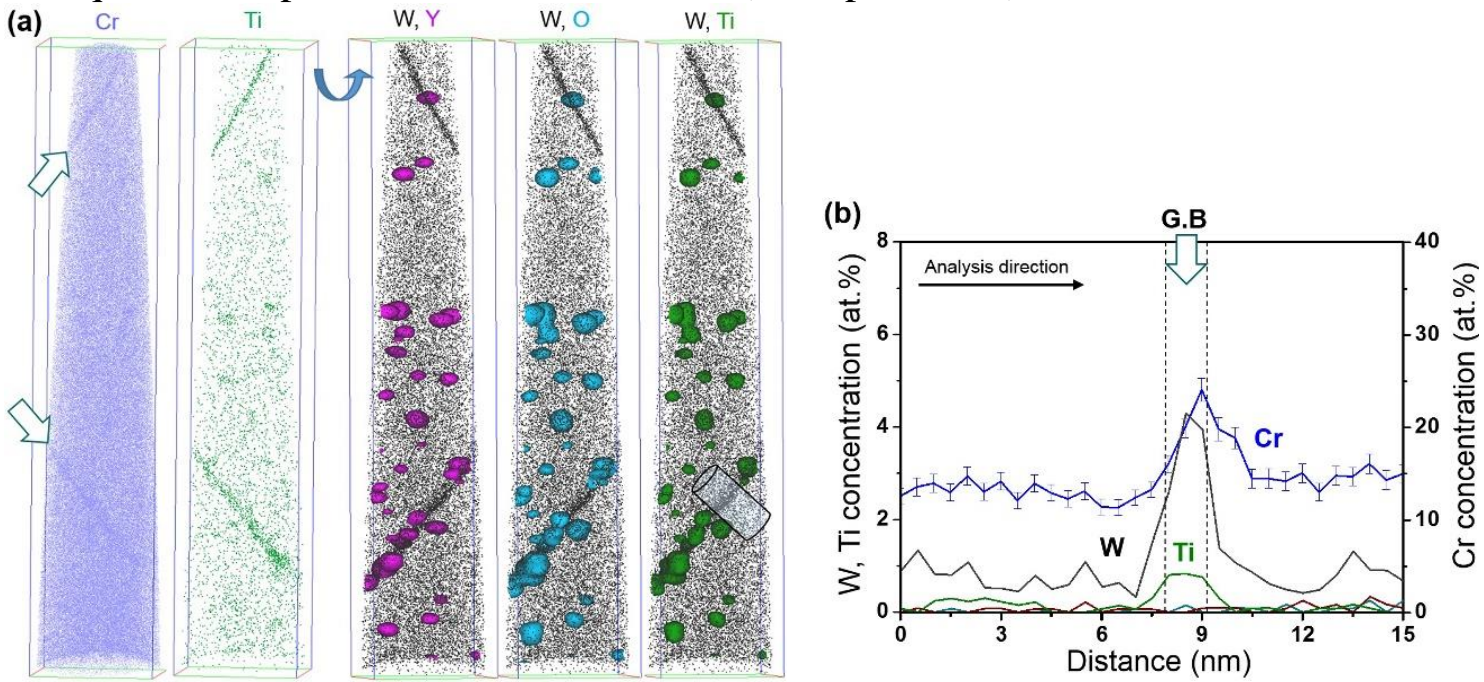

Figure 1. (a) Morphology and arrangement of NFs: reconstructed APT maps of Cr (blue) and Ti (green). $\mathrm{W}$ (black) and NFs are visualized with a iso-concentration surface of 0.7 at.\% Y (pink), 2.0 at.\% O (sky blue), and 2.0 at.\% Ti; (b) $1 \mathrm{D}$ concentration profiles along the cylinder ( $\Phi=10 \mathrm{~nm})$ in (a) with a bin size of $0.4 \mathrm{~nm}$.
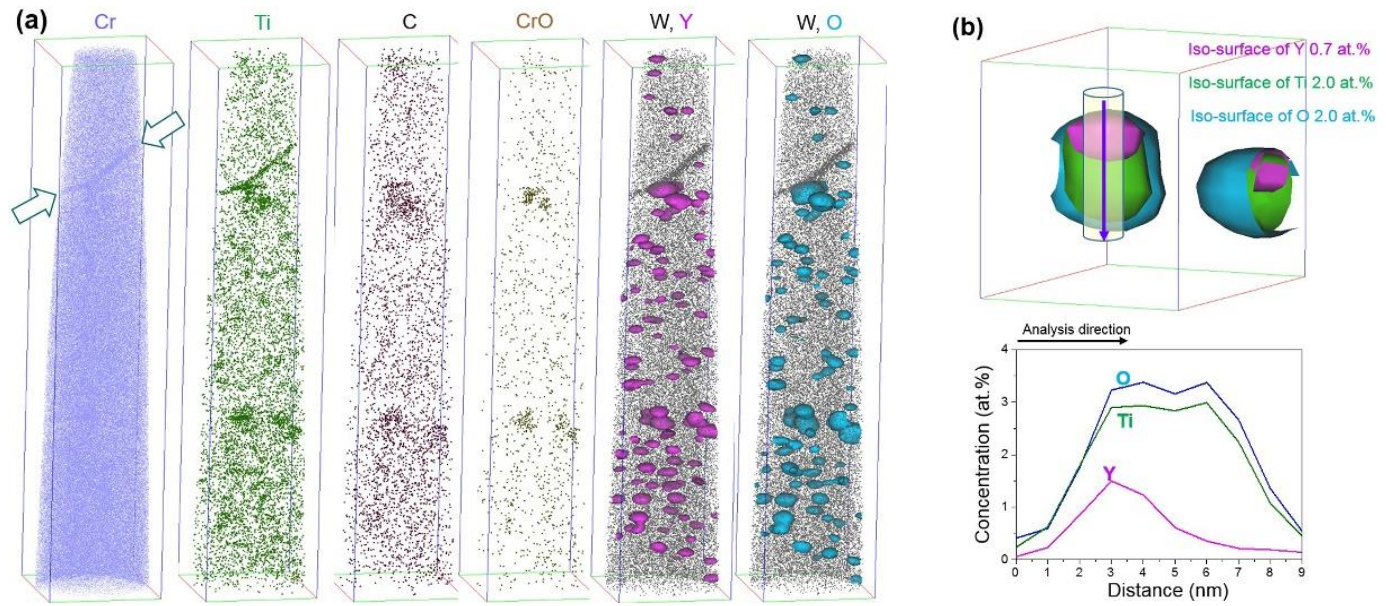

Figure 2. (a) Reconstructed APT maps of Cr (blue), Ti (green), C ( ), CrO (gold), and W (black) with visualization of NFs; (b) 1D concentration profile with respect to interfaces of NFs, calculated with a bin size of $0.4 \mathrm{~nm}$. 